\title{
Immunological Features of Surgical Stress in Underage and Adult Patients
}

Fishman $\mathrm{B}^{1}$, Tapbergenov $\mathrm{T}^{1}$, Kuprin $\mathbf{P}^{1}$, Turmakhanov $\mathbf{S}^{1,3}$, Saydayev Z1,3, Kushpita A², Martynov Iㅁ, Karasev D', Yukhno $\mathbf{M}^{4}$ and Rumyantsev $\mathrm{Y}^{1 *}$

${ }^{1}$ Yaroslav-the-Wize Novgorod State University, Institute of Medical Education, Veliky Novgorod, Russia

${ }^{2} \mathrm{~S}$ M Kirov Military Medical Academy, Saint Petersburg, Russia

${ }^{3}$ Regional Clinical Hospital, Veliky Novgorod, Russia

${ }^{4}$ Central City Clinical Hospital, Veliky Novgorod, Russia

*Corresponding author: Yegor Rumyantsev, Yaroslav-the-Wize Novgorod State University, Institute of Medical Education, Veliky Novgorod, Russia, Email: gogathejedi@gmail.com

\section{Abstract}

Already in the 60-70s of the last century, it was firmly established that, regardless of the initial general condition of the patient and the level of his immunoreactivity, the surgical operation itself has an adverse effect on the immune system and causes the development of immunodeficiency. In the phagocytic system of immunity, under the influence of surgical intervention, both quantitative (lowering the number of main phagocytic cells: neutrophils and monocytes) and qualitative changes (lowering the ability of phagocytes to capture, kill and digest microorganisms) are observed. It is important to note that during the operation, the population of phagocytic cells that is closest to the site of intervention is more affected. Cells of the monocyte-macrophage system play an important role in the induction of both cellular and humoral immune response. Changes in humoral immunity consist in lowering blood level of all classes of immunoglobulins (IgG, IgA, IgM) and especially IgG - the main component of humoral immunity. In the cellular immunity, under the influence of surgery, quantitative and qualitative changes are also observed. First of all, the total number of lymphocytes decreases and the level of T-lymphocytes decreases while maintaining the ratio between the two main subpopulations: T-helpers and T-suppressors.

Cytokines play a major role in the implementation of the inflammatory response to surgical trauma. The local release of cytokines - interleukin-1 (IL-1), -6 (IL-6), tumor necrosis factor (TNF) coordinates the local inflammatory response at the site of injury, inducing neutrophil chemotaxis to the area of inflammation. Cytokines induce fever and acute phase reactions, mobilize neutrophils from the bone marrow and cause lymphocyte proliferation. The action of cytokines is 


\section{Virology \& Immunology Journal}

complex, to a certain extent, interdependent. Cytokine production reflects on the invasiveness of surgery. So, it is minimal in laparoscopic procedures and maximal in prosthetic joints, large vascular and abdominal operations.

Keywords: Surgical Stress; Cytokines; Immunology; Neuroendocrine System

Abbreviations: TNF: Tumor Necrosis Factor; IL: Interleukin; PGE2: Prostaglandin E2; HF: High-Frequency Waves; LF: Low-Frequency Waves.

\section{Introduction}

According to Chaitov R, et al. already in the 60-70s of our century it was firmly established that, regardless of the initial general condition of the patient and the level of his immunoreactivity, the surgical operation itself has an adverse effect on the immune system and causes the development of immunodeficiency, the main manifestation of which are infectious postoperative complications [1-3]. According to many authors, in the phagocytic system of immunity under the influence of surgical intervention, both quantitative and qualitative changes are observed [1,2,4-6]. Quantitative changes consist in decrease in number of the main phagocytic cells: neutrophils and monocytes. Qualitative changes consist in lowering the ability of phagocytes to capture, kill and digest microorganisms. The decrease in functional properties of phagocytes is at least partially related to the suppression of their ability to form reactive oxygen species: superoxide anion, hydroxyl radical, singlet oxygen, etc., which play an extremely important role in the oxygen-dependent killing of microorganisms $[4,5]$.

Cells of the monocyte-macrophage system play an important role in the induction of both cellular and humoral immune response. Surgery disrupts this function of monocytes / macrophages: on the 2nd and 7th days after surgery, a significant decrease in the expression of HLA-DR and HLA-DQ antigens on these cells occurs, resulting in a reduced ability of the organism to develop a specific immune response [7].

Changes in humoral immunity consist in decreasing blood level of all classes of immunoglobulins (IgG, IgA, IgM), especially the main component of humoral immunity - IgG [7,8]. In the cellular immunity, quantitative and qualitative changes are also observed. First of all, the total number of lymphocytes decreases and the level of T-lymphocytes decreases while maintaining the ratio between the two main subpopulations: T-helpers and T-suppressors, and similar results were obtained in analyzing the immune status both in the experiment and in the clinic [7-9]. The authors note that after surgery there is a sharp decrease in the total population of $T$ (CD3) lymphocytes, $T$ (CD4) helper cells, T (CD8) suppressors and B lymphocytes in both peripheral blood and spleen and mesenteric lymph node [9]. The decrease in the functional activity of T-cells after surgery is also manifested in the reduced ability of these cells to give a proliferative response to T-mitogens and in the ability to synthesize some cytokines [10]. So, there is a significant decrease in the ability of T-cells to produce one of the central cytokines of the immune system, IL-2 [11]. One of the reasons for this decrease is the appearance of a soluble receptor for IL-2 (IL-2P) in the serum, which is one of the potent inhibitors of IL-2. The latter appears in the serum of patients on the 1st day and reaches a peak on the 3rd day after the operation. By this time, there is also a maximum decrease in the ability to produce IL-2 in the peripheral blood lymphocytes of the operated patients. The degree and duration of this decrease depends on the duration of the operation, the size of the wound $[11,12]$. One of the reasons for the emergence of IL-2R may be an increase in peripheral blood of operated patients with the number of activated T-lymphocytes observed in some operations, characterized by the presence of activation markers on the surface membrane: HLA-DR antigens and an IL-2 receptor [13]. This increase can probably be regarded as the body's response to the inflammatory process, which to some extent always develops after surgery. As a result of dropping (shedding) IL-2P from the surface of activated T-lymphocytes, it appears in increased amounts in the blood and has a suppressive effect on T-lymphocytes. In most cases, the maximum immunodepression in almost all parameters of the immune system is observed on the 2nd day after the operation and depending on the nature of this operation and the initial state of the patient, its duration varies from 7 to 28 days $[3,14]$.

At present, some of the main causes of the development of immunodeficiency states after surgery are becoming clear. One of these causes is a disorder in the body under the influence of the operation of immunoregulatory processes carried out with the help of TH1- and TH2-helpers. The first synthesize cytokines that 


\section{Virology \& Immunology Journal}

stimulate cellular immunity ( $\gamma$-interferon, TNF- $\alpha$, IL-1 $\beta$, etc.), the second synthesize cytokines that stimulate humoral immunity (IL-4, IL-10, TRF, etc.). Cytokines synthesized by TH1 cells inhibit the function of TH2 cells, and vice versa $-\gamma$-interferon, TNF- $\alpha$ and IL- $1 \beta$, as well as IL-12, produced by macrophages, are potent activators of T-lymphocytes and phagocytic system cells (like neutrophils, and monocytes / macrophages). Their antagonists are IL-4, IL-10 and TRF: these cytokines inhibit cellular immunity and, therefore, the functional activity of phagocytic cells.

Thus, an increase in the functional activity of $\mathrm{TH} 2$ helpers and a decrease in the functional activity of TH1helpers is one of the main reasons for the development of surgical infections. But a significant increase in the activity of the opposed subpopulation of lymphocytes TH1-helper cells leads to the development of other, equally serious complications. As just noted, these cells synthesize IL-1 $\beta$ and TNF- $\alpha$. Note that these proinflammatory cytokines are also the main factors in the development of septic shock. Indeed, it has been shown that a decrease in the functional activity of the TH2-helper cells and the level of cytokines produced by them is one of the leading causes of the development of septic shock. A special role here belongs to IL-10, which is the strongest inhibitor of the synthesis of pro-inflammatory cytokines by macrophages - IL-1 and TNF [15]. In addition to cytokines, prostaglandin E2 (PGE2) plays a certain role in the inhibition of TH1-helper cells and, consequently, the cellular immune response. Under the influence of surgery, monocytes / macrophages are activated, which manifest in the enhancement of their synthesis of PGE2. The latter is a potent immunosuppressive agent: it inhibits the proliferative activity of T-lymphocytes and the synthesis of a number of cytokines and, above all, IL-2. It also suppresses the functional activity of B-lymphocytes. In addition, PGE2 inhibits the function of TH1 lymphocytes and activates the functional activity of TH2 cells with the synthesis of immunosuppressive cytokines [6]. The inhibitory effect on the immune system and other factors formed in the body under the influence of surgery must be taken into account. Surgery is stress, and under stress, as will be shown in more detail below, there is an increased release of corticosteroids, catecholamines, endorphins, etc. into the bloodstream. All of them are far from being indifferent to the body's immune system and each of them makes its own negative contribution to the functioning of this system after surgery. In general, surgery has a complex and multifaceted effect on the immune system, which can be characterized as an activation and suppression. This is a result of the fact that the operation can act differently on the opposite functioning immunoregulatory cells: activation of the function of TH2 cells leads to the development of surgical infections; activation of the function of TH1 cells leads to the development of septic shock.

Therefore, the whole complex of changes that occurs in the immune system under the influence of the operation can be briefly described as deregulation of immunity [16-18].

\section{Methods}

Study group consisted of 57 underage patients and 64 adult patients with a traumatic disease of varying severity who were treated promptly for the underlying disease. The average age of children was $10.5 \pm 2.5$ years, the average age of adults was $42.5 \pm 11.5$ years. A comprehensive examination was carried out in the preoperative period for 1 day before surgery, in the intraoperative period on the day of surgery, and in the postoperative period for 3-5 days after surgery. The nature, duration of surgery and type of anesthesia were not taken into account. The control group consisted of practically healthy children and adults of similar age.

A study of thyroid function was performed by determining blood level of thyroid hormones: thyroxine (T4), triiodothyronine (T3), and also thyroid-stimulating hormone of the pituitary (TSH) using the radionuclide method using standard test kits from Boehringer Ingelheim.

The study of the immune system was performed by flow cytometry, and the total numbers of lymphocytes, Tlymphocytes (CD3 +) and B-lymphocytes (CD72 +) were counted in the peripheral blood. Subpopulations of T-cells were predominantly determined with suppressor and helper activity, including theophylline-sensitive rosetteforming cells (TRFC-sens) and theophylline-resistant rosette-forming cells (TRFC-res), the number of circulating immune complexes, phagocytic activity.

In our study, we performed a spectral analysis of heart rate variability (HRV) after recording an ECG of at least 100 R-R intervals using an EK12K-01 cardioanalyzer and the Kardis 2.0 software package. The relative values of TF, $\mathrm{LF}, \mathrm{HF}$ and the ratio of LF / HF were calculated.

StatSoft Statistica software, ver. 10.0.A was used for parametric and nonparametric calculations. Statistical significance was stated at $\mathrm{p}<0,05$. 


\section{Virology \& Immunology Journal}

\section{Results}

Despite the psychoemotional stress, in the preoperative period of surgical stress, the optimal values of the vagosympathetic balance ( $\mathrm{LF} / \mathrm{HF} \approx 1$ ) were noted, which is reflected in Table 1.

\begin{tabular}{|c|c|c|c|}
\hline \multirow{2}{*}{ Indicators } & \multicolumn{3}{|c|}{ Surgical stress period } \\
\cline { 2 - 4 } & Preoperative & Intraoperative & Postoperative \\
\hline TF & $3725 \pm 502$ & $4123 \pm 543^{*}$ & $3734 \pm 491^{*}$ \\
\hline LF & $1225 \pm 85$ & $1638 \pm 92^{*}$ & $1322 \pm 71^{*}$ \\
\hline HF & $1200 \pm 89$ & $876 \pm 53^{*}$ & $1093 \pm 67^{*}$ \\
\hline LF/HF & 1,02 & $1,87^{*}$ & $1,21^{*}$ \\
\hline
\end{tabular}

Note: $\left({ }^{*}\right)$ - statistically significant differences $(\mathrm{p}<0.05)$ compared with the previous period.

Table 1: Indicators of vagosympathetic balance in adults with surgical stress ( $\mathrm{ms}^{2}, \mathrm{M} \pm \mathrm{SD}$ ).

In the intraoperative period, the total spectral power (TF) increased by $10.7 \%$ in adults. Moreover, this happened mainly due to low-frequency waves (LF), whose power increased by $33.7 \%$ compared with the preoperative stage $(\mathrm{p}<0.05)$. The power of highfrequency waves (HF) during surgery decreased by $27.0 \%$, which led to a shift in the vagosympathetic balance towards the predominance of the sympathetic effect on HRV (LF / HF> 1.5).

In the postoperative period, changes in the spectral indices of HRV tended to restore their original values in adults. However, unlike that in children, the power of low- frequency waves (LF) maintained elevated values compared with baseline $(\mathrm{p}<0.05)$, although it decreased by $19.3 \%$ compared with the intraoperative period. This can be explained by the preservation of sympathetic impulses in the postoperative period in adults, despite the fact that the values of the vagosympathetic balance coefficient indicate a slight decrease in the sympathetic effect on HRV.

In adults, in the preoperative period of surgical stress, a balanced ratio adrenaline/noradrenaline (A/NA) in urine is observed Table 2 .

\begin{tabular}{|c|c|c|c|}
\hline \multirow{2}{*}{ Indicators } & \multicolumn{3}{|c|}{ Surgical stress period } \\
\cline { 2 - 4 } & Preoperative & Intraoperative & Postoperative \\
\hline A & $6,85 \pm 0,85$ & $17,85 \pm 2,25^{*}$ & $9,68 \pm 1,76^{*}$ \\
\hline NA & $6,61 \pm 0,76$ & $13,67 \pm 3,33^{*}$ & $8,76 \pm 2,21^{*}$ \\
\hline A/NA & 1,04 & $1,30^{*}$ & $1,10^{*}$ \\
\hline
\end{tabular}

Note: $\left({ }^{*}\right)$ - statistically significant differences $(\mathrm{p}<0.05)$ compared with the previous period

Table 2: The daily excretion of catecholamines in the urine in adults under surgical stress (ng/ml, $\mathrm{M} \pm \mathrm{SD}$ ).

In the intraoperative period, in adults as well as in children, there is an increase in urinary catecholamine excretion $(\mathrm{p}<0.05)$, with the level of adrenaline rising 2.6 times, and norepinephrine -2.1 times. The A/NA ratio shifts towards the predominance of the adrenal regulation $(\mathrm{A} / \mathrm{NA}>1)$.

In the postoperative period in adults, the excretion of catecholamines in urine decreases $(p<0.05)$, however, it remains above the preoperative level: adrenaline - by $41.3 \%$, and noradrenaline - by $32.5 \%$. The A / HA ratio indicated a slight predominance $(\mathrm{p}<0.1)$ of the adrenal regulation.

Thus, the data from this study indicate a significant activation of the sympathoadrenal system during surgical stress, which is manifested in an increase in the basal excretion of catecholamines in the urine. However, in underage patients, sympathoadrenal system activation leads to a subsequent depletion of sympathetic impulses in the postoperative period, which is manifested in a decrease in the excretion of noradrenaline in the urine and an increase in the A/NA ratio. Apparently, intrasystem imbalance occurs, which characterizes the state of disadaptation of the child's body during surgical stress.

At the same time, in adults, study of the density of receptors for norepinephrine on lymphocytes by rosette method showed an increase in the number of rosetteforming cells (RFC) with bull erythrocytes sensitized with norepinephrine in the intraoperative (by 20.6\%) and 


\section{Virology \& Immunology Journal}

postoperative (by 12\%) periods of surgical stress Table 3 was found.

\begin{tabular}{|c|c|c|c|}
\hline \multirow{2}{*}{ Indicators } & \multicolumn{3}{|c|}{ Surgical stress period } \\
\cline { 2 - 4 } & Preoperative & Intraoperative & Postoperative \\
\hline RFC number & $9,2 \pm 0,5$ & $11,1 \pm 0,3^{*}$ & $10,3 \pm 0,7^{*}$ \\
\hline
\end{tabular}

Note: $\left({ }^{*}\right)$ - statistically significant differences $(\mathrm{p}<0.05)$ compared with the previous period

Table 3: The number of RFC with norepinephrine in adults with surgical stress (per 100 cells, $\mathrm{M} \pm \mathrm{SD}$ ).

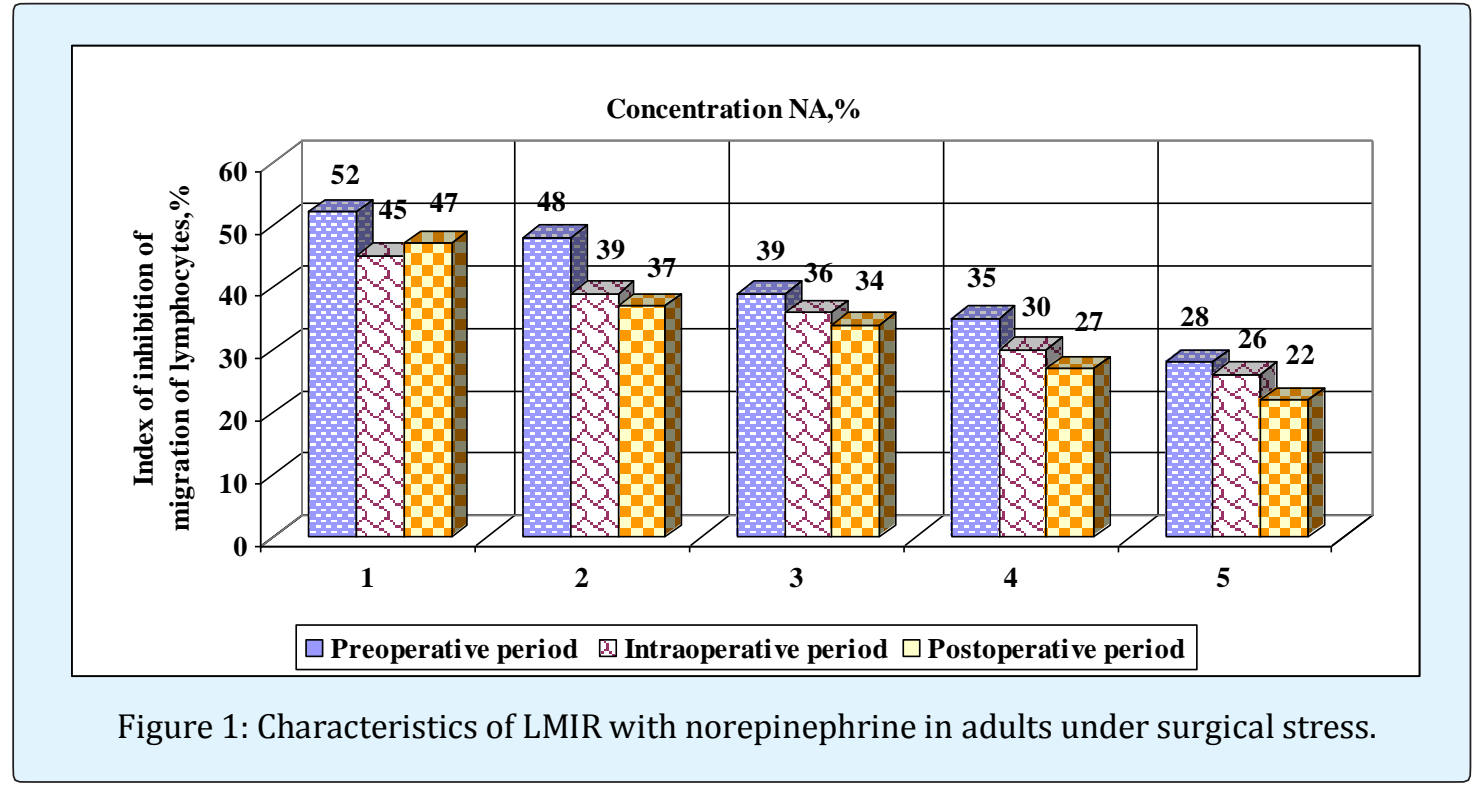

Studying receptor sensitivity to noradrenaline, an analysis of the results of leukocyte migration inhibition reaction (LMIR) with the mitogen Con $A$ in the presence of different concentrations of noradrenaline showed that during the intraoperative and postoperative periods of surgical stress, the lymphocyte migration index decreases in response to a decrease in the noradrenaline concentration in the incubation medium (Figure 1).

These data may indicate a decrease in receptor sensitivity to norepinephrine on lymphocytes in adults.

\begin{tabular}{|c|c|c|c|}
\hline \multirow{2}{*}{ Indicators } & \multicolumn{3}{|c|}{ Surgical stress period } \\
\cline { 2 - 4 } & Preoperative & Intraoperative & Postoperative \\
\hline $\mathrm{TSH}, \mathrm{mU} / \mathrm{l}$ & $2,72 \pm 0,24$ & $3,76 \pm 0,36^{*}$ & $3,45 \pm 0,30$ \\
\hline $\mathrm{T} 3, \mathrm{nmol} / \mathrm{l}$ & $2,63 \pm 0,23$ & $2,12 \pm 0,18^{*}$ & $2,87 \pm 0,21^{*}$ \\
\hline $\mathrm{T} 4, \mathrm{nmol} / \mathrm{l}$ & $123,3 \pm 7,3$ & $117,5 \pm 5,5^{*}$ & $145,5 \pm 9,7^{*}$ \\
\hline $\mathrm{T} 3+\mathrm{T} 4 / \mathrm{TSH}$ & 46,3 & $31,8^{*}$ & $43,0^{*}$ \\
\hline $\mathrm{T} 3 / \mathrm{T} 4{ }^{\prime} 100 \%$ & 2,13 & $1,80^{*}$ & $1,97^{*}$ \\
\hline
\end{tabular}

Note: $\left({ }^{*}\right)$ - statistically significant differences $(\mathrm{p}<0.05)$ compared with the previous period.

Table 4: Blood level of thyroid hormones in underage patients with surgical stress $(\mathrm{M} \pm \mathrm{SD}$ ).

In the study of blood level of thyroid hormones in underage patients with surgical stress (Table 4), a significant increase $(\mathrm{p}<0.05)$ in the TSH level by $38.2 \%$ in the intraoperative period is noted. At the same time, the level of T3 and T4 decreased significantly $(\mathrm{p}<0.05)$ by $19.4 \%$ and $4.7 \%$, respectively. In addition, indicators of thyroid activity: the thyroid index (T3 + T4 / TSH) and the 


\section{Virology \& Immunology Journal}

ratio T3 / T4 also significantly decreased, which indicates an inhibition of thyroid function during surgical stress.

In adults with surgical stress (Table 5), a significant increase in TSH ( $p>0.05$ ) was observed in the intraoperative and postoperative periods by $52.6 \%$ and
$24.3 \%$, respectively. At the same time, the level of T3 and $\mathrm{T} 4$ decreased by $48.7 \%$ and $18.9 \%$, respectively. A decrease in thyroid activity is also indicated by a decrease in the thyroid index (by 47.2\%) and the ratio T3 / T4 (by $36.9 \%)$.

\begin{tabular}{|c|c|c|c|}
\hline \multirow{2}{*}{ Indicators } & \multicolumn{3}{|c|}{ Surgical stress period } \\
\cline { 2 - 4 } & Preoperative & Intraoperative & Postoperative \\
\hline $\mathrm{TSH}, \mathrm{mU} / \mathrm{l}$ & $1,75 \pm 0,15$ & $2,67 \pm 0,23^{*}$ & $3,32 \pm 0,32^{*}$ \\
\hline $\mathrm{T} 3, \mathrm{nmol} / \mathrm{l}$ & $1,99 \pm 0,13$ & $1,02 \pm 0,12^{*}$ & $2,45 \pm 0,15^{*}$ \\
\hline $\mathrm{T} 4, \mathrm{nmol} / \mathrm{l}$ & $91,5 \pm 5,5$ & $74,2 \pm 8,4^{*}$ & $93,3 \pm 6,3^{*}$ \\
\hline $\mathrm{T} 3+\mathrm{T} 4 / \mathrm{TSH}$ & 53,4 & $28,2^{*}$ & 28,8 \\
\hline $\mathrm{T} 3 / \mathrm{T} 4$ ' $100 \%$ & 2,17 & $1,37^{*}$ & $2,63^{*}$ \\
\hline
\end{tabular}

Note: $\left({ }^{*}\right)$ - statistically significant differences $(\mathrm{p}<0.05)$ compared with the previous period Table 5: Blood level of thyroid hormones in adults with surgical stress (M \pm SD).

During postoperative period, in adults, while the level of TSH increased, the T3 level significantly increased, so that its level even exceeded the preoperative level by $23.1 \%$. At the same time, when studying thyroid activity, the changes did not correlate: the thyroid index remained low, and the $\mathrm{T} 3$ / T4 ratio increased by $92.0 \%$ compared with the intraoperative period $(\mathrm{p}<0.05)$.

As a result of a study of thyroid function, it was established that during surgical stress, despite an increase in blood level of TSH, a depression of the function of the thyroid system is observed in both children and adults. In the postoperative period in adults and children, blood level of thyroid hormones increases. However, in adults, thyroid activity is restored faster, which may indicate a higher adaptive capacity of the thyroid system in adults than in children.

\section{Summary}

Results of our study indicate that during surgical stress, significant changes in the adrenal-thyroid system are observed, manifested in increased sympathetic activity, which in children, unlike adults, can lead to depletion of sympathetic impulses in the postoperative period. At the same time, in adults, the density of norepinephrine receptors on lymphocytes increases and their sensitivity decreases. At the same time, in spite of the increased blood level of TSH, in adults, surgical stress causes a suppression of the function of the thyroid system, manifested in a decrease of blood level of T3 and T4. In the postoperative period, blood level of thyroid hormones in children and adults increases. However, in adults, thyroid activity is restored faster than in children, which may indicate a higher adaptive capacity of the thyroid system of the adult organism.

\section{References}

1. Chaitov R, Pinegin B (1998) Ismenenie immuniteta pri chirurgicheskich vmeiatelstvach. Annali chirurgicheskoi gepatologii Tom 3(2): 100-110.

2. Solov'ev G, Petrova I, Kovalev S (1987) Immunokorrekciia, profilactica i lechenie gnoinosepticheskich zabolevanii pp: 145.

3. Lennard TW, Shenton BK, Borzotta A, Donnelly PK, White $\mathrm{M}$, et al. (1985) The influence of surgical operations on components of the human immune system. BrJ Surg 72(10): 771-776.

4. Belotskii S (1985) Effekt chirurgii na fagocitarnuu sistemu bolnich. Chirurgia 2: 92-94.

5. Chaitov R, Pinegin B, Butakov A, Andronova T (1994) Immunotherapy of infectious postoperative complications with glucosaminylmuramyl dipeptide. Immunotherapy of Infectious pp: 205-211.

6. Saba TM, Di Luzio NR (1969) Surgical stress and reticuloendothelial function. Surgery 65(5): 802-807.

7. Ryhanen P, Surcel HM, Ilonen J (1991) Decreased expression of class II major histocompatibility complex (MHC) molecules on monocytes is found in open-heart surgery related immunosuppression. Acta Anaesthesiol Scand 35(5): 453-456. 


\section{Virology \& Immunology Journal}

8. Roge HN, Christou NV, Buhenick O, Superina R, Gordon J, et al. (1982) Lymphocyte function in anergic patients. Clin Exp Immunol 47(1): 151-161.

9. Barrena MJ, Eizaguirre I, Aldazabal P, Cuadrado E, Bachiller P, et al. (1995) Lymphocyte subpopulations after extensive small bowel resection in the rat. J Pediatr Surg 30(10): 1447-1449.

10. Zellweger R, Ayala A, DeMaso CM, Chaudry IH (1995) Trauma-hemorrhage causes prolonged depression in cellular immunity. Shock 4(2): 149-153.

11. Lahat N, Shtiller R, Zlotnick AY, Merin G (1993) Early IL-2/slL-2R surge following surgery leads to temporary immune refractoriness. Clin Exp Immunol 92(3): 482-486.

12. Delogu G, Reale G, Marchei GG, Rezaeieh RH, Casula MA, et al. (1992) Neopterin and interleukin 2 soluble receptors as biochemical markers of cellular immune response to surgical trauma. Ann Ital Chir 63(3): 359362.

13. Mizutani Y, Terachi T, Okada Y, Yoshida O (1996) Effect of surgical stress on immune function in patients with urologic cancer. Int J Urol 3(6): 426434.
14. Roth JA, Golub SH, Grimm EA, Eilber FR, Morton DL (1976) Effects of operation on immune response in cancer patients: sequential evaluation of in vitro lymphocyte function. Surgery 79(1): 46-51.

15. Ertel W, Keel M, Steckholzer U, Ungethüm U, Trentz 0 (1996) Interleukin-10-attenuates he release of proinflarnmatory cytokines but depresses splenocyte functions in murine endotoxemia. Arch Surg 131(1): 51-56.

16. Braga M, Vignali A, Gianotti L, Cestari A, Profili M, et al. (1996) Immune and nutrition-al effects of early enteral nutrition after major abdominal operations. Eur J Surg 162(2): 105-112.

17. Brand JM, Kirchner H, Poppe C, Schmucker P (1997) The effects of general anesthesia on human peripheral immune cell distribution and cytokine production. Clin 1m-munol Immunopathol 83(2): 190-194.

18. Chaundry IH, Ayala A (1993) Mechanism of increased susceptibility to infection following hemorrhage. Am J Surg 165(2): 59-67.

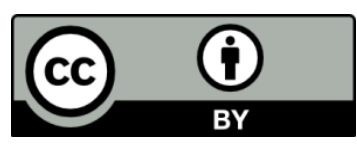

Rumyantsev Y, et al. Immunological Features of Surgical Stress in Underage and Adult Patients. Virol Immunol J 2018, 2(11): 000198. 